\title{
3D-QSAR Studies of 1,2,4-Oxadiazole Derivatives as Sortase A Inhibitors
}

\author{
Neda Shakour, ${ }^{1,2}$ Farzin Hadizadeh (iD, ${ }^{1,3}$ Prashant Kesharwani, ${ }^{4}$ \\ and Amirhossein Sahebkar $\mathbb{D D}^{3,5,6}$ \\ ${ }^{1}$ Department of Medicinal Chemistry, School of Pharmacy, Mashhad University of Medical Sciences, Mashhad, Iran \\ ${ }^{2}$ Student Research Committee, Faculty of Medicine, Mashhad University of Medical Sciences, Mashhad, Iran \\ ${ }^{3}$ Biotechnology Research Center, Pharmaceutical Technology Institute, Mashhad University of Medical Sciences, Mashhad, Iran \\ ${ }^{4}$ Department of Pharmaceutics, School of Pharmaceutical Education and Research, Jamia Hamdard, New Delhi 110062, India \\ ${ }^{5}$ Applied Biomedical Research Center, Mashhad University of Medical Sciences, Mashhad, Iran \\ ${ }^{6}$ Department of Biotechnology, School of Pharmacy, Mashhad University of Medical Sciences, Mashhad, Iran
}

Correspondence should be addressed to Farzin Hadizadeh; hadizadehf@mums.ac.ir and Amirhossein Sahebkar; amir_saheb2000@yahoo.com

Received 2 August 2021; Revised 23 October 2021; Accepted 13 November 2021; Published 6 December 2021

Academic Editor: Muhammad Farrukh Nisar

Copyright ( $) 2021$ Neda Shakour et al. This is an open access article distributed under the Creative Commons Attribution License, which permits unrestricted use, distribution, and reproduction in any medium, provided the original work is properly cited.

\begin{abstract}
Sortase A (SrtA) is an enzyme that catalyzes the attachment of proteins to the cell wall of Gram-positive bacterial membrane, preventing the spread of pathogenic bacterial strains. Here, one class of oxadiazole compounds was distinguished as an efficient inhibitor of SrtA via the "S. aureus Sortase A" substrate-based virtual screening. The current study on 3D-QSAR was done by utilizing preparation of the structure in the Schrödinger software suite and an assessment of 120 derivatives with the crystal structure of 1,2,4-oxadiazole which was extracted from the PDB data bank. The docking operation of the best compound in terms of pMIC (pMIC = 2.77) was done to determine the drug likeliness and binding form of 1,2,4-oxadiazole derivatives as antibiotics in the active site. Using the kNN-MFA way, seven models of 3D-QSAR were created and amongst them, and one model was selected as the best. The chosen model based on $q^{2}$ (pred_r $r^{2}$ ) and $R^{2}$ values related to the sixth factor of PLS illustrates better and more acceptable external and internal predictions. Values of crossvalidation (pred_ $r^{2}$ ), validation $\left(q^{2}\right)$, and $F$ were observed $0.5479,0.6319$, and 179.0 , respectively, for a test group including 24 molecules and the training group including 96 molecules. The external reliability outcomes showed that the acceptable and the selective 3DQSAR model had a high predictive potential $\left(R^{2}=0.9235\right)$ which was confirmed by the $Y$-randomization test. Besides, the model applicability domain was described successfully to validate the estimation of the model.
\end{abstract}

\section{Introduction}

Sortase A (SrtA) is a polypeptide containing 206 amino acids. This enzyme speeds up two consecutive reactions: (a) transpeptidation and (b) thioesterification. SrtA is involved in the bacterial adhesion process and acts by attaching proteins holding LPXTG to lipid II [1-11]. SrtA inhibitors do not influence bacterial growth, but instead, they prevent the emergence of the virulence of pathogenic bacterial strains, thereby hindering infections produced by Staphylococcus aureus (S. aureus) or other bacteria of
Gram-positive strain. To the surface membrane protein of S. aureus, sortase is attached which links it to the cell wall via transpeptidation $[6,10,12]$, and needing a C-terminal regulates signal through a protected LPXTG motif [13-15]. $S$. aureus mutations with a deficiency of the srtA gene cannot display and bind some surface proteins which results in a disorder/disease such as animal infection $[13,16]$. $S$. aureus is an important anthropological bacterial pathogen of Gram-positive strain that leads to common infections in society. Regarding the resistance to antibiotics, and the report of the Centers for Disease Control and Prevention 
(CDC) about resistance to methicillin of S. aureus (MRSA) in 2013, S. aureus was distinguished as a critical and a persistent threat [17-19].

The Gram-positive bacteria $S$. aureus is communal to humans and exists on the mucosa and skin of $30 \%$ of the population $[20,21]$. It is a chief reason for hospital infections, the most common and serious of which are endocarditis and bacteremia endocarditis in hospitalized patients [22-25]. This organism has created resistance to a broad range of antibiotic medicine types [26]. Principal commercial compounds of the antimicrobial class (such as ciprofloxacin, ampicillin, and posaconazole) have limited performance against resistance microorganisms strains [27-30]. The erratic usage of antibiotics is known as one of the primary reasons for the increase in resistance of bacteria. The growth of bacterial resistance has resulted in a significant rise in mortality rates of individuals around the world [31]. On this path, there is a serious necessity to discover novel molecules with more effective antibacterial features, as well as obvious synthetic routes. This led to widespread research such as designing heterocyclic derivatives (like 1,2,4-oxadiazoles) with antimicrobial properties to treat $S$. aureus infections [32-36]. These discovered antibiotics are active and exhibit grampositive activity, particularly against Staphylococcus aureus, including vancomycin-resistant, methicillinresistant (MRSA), and linezolid-resistant by inhibiting srtA [24, 35, 37]. 1,2,4-Oxadiazole heterocycle was first manufactured in 1884. They showed remarkable action in vitro and in vivo and are orally bioavailable. The medicinal chemistry literatures report diverse structures for the 1,2,4-oxadiazoles (Figure 1) [38, 39]. In the present article, we represent 3D-QSAR investigations concerning $120 \mathrm{~mol}-$ ecules of 1,2,4-oxadiazoles with antibacterial healing properties. This class of compounds (oxadiazoles) targets SrtA of the cell wall and inhibits it [40]. The advancement of antibiotics, especially of those that target cells of bacteria and have a desirable characteristic of toleration and safety, has largely helped population growth and has improved the quality of life in the last 75 years.

\section{Materials and Methods}

2.1. Data Set. A collection containing 120 compounds having 1,2,4-oxadiazole as antimicrobials was taken from the available literature [24] and was employed in the present study. All structures were extracted from Chembl (https://www.ebi.ac.uk/chembldb). The chosen compounds for the set of data shared a similar evaluation method with notable changes in their strength profiles and their structures. The compounds incorporated in the collection of datasets have antibiotics potencies with MIC values varying from 2 to $500 \mu \mathrm{g} / \mathrm{ml}$ which were changed to $\mathrm{M}$ (molar). These were then converted to pMICs according to the following equation [41-44].

$\mathrm{pMIC}=-\log _{10}[\mathrm{MIC}]$.

The ligand 3D-formula of compounds was produced utilizing the Maestro v2015-2 and afterwards corrected using the LigPrep. Partial charges of atoms were attrib- uted, and potential ionization was calculated at a neutral medium. The force field of OPLS_2005 was utilized for minimization to the conformer creation with the low energy of ligand. The minimization of energy was done for every compound (ligand) to reach an RMSD cutoff of $0.01 \AA$. Then, the final structures were used for modeling investigation. Additionally, all the 120 molecules were aligned in the method of alignment (Figure 2) relying on Maestro through choosing a common structure minimum as "template" and the most efficient one (compound 89) as the "Reference Molecule" (Figure 3). Of the 120 molecules recognized in this investigation, a training group, including 96 molecules and a test group, including 24 molecules was created in Maestro [45-48].

2.2. Model Validation and Statistical Analysis. A high $q^{2}$ only indicates a good internal validation in the training group, but it does not show a high prediction capability of the created models; hence, an external validation was necessary. The proved capability from generated models of 3D-QSAR was confirmed by computing the biological activities of compounds that applied as a test set and not inserted in the training set (Suppl. Table 1). In the present investigation, eighty percent of the molecules from the data set was accidentally chosen as training set models based on the atom field which were created of PLS factors (one until seven), and the obtained models were approved after predicting the activity of the test group ligand. The value of the model's prediction was assessed through the leaveone-out (LOO). The $q^{2}$ (predictive correlation coefficient) was determined by utilizing Equation (1) [49].

$$
q^{2}=1-\frac{\sum\left(Y_{\text {predicted }}-Y_{\text {observed }}\right)^{2}}{\sum\left(Y_{\text {observed }}-Y_{\text {mean }}\right)^{2}} .
$$

In the above equation, each of the three indices including $Y_{\text {mean }}, Y_{\text {predicted }}$, and $Y_{\text {observed }}$, demonstrates the mean values predicted and observed of the pMIC feature, respectively. The $\left(Y_{\text {predicted }}-Y_{\text {observed }}\right)^{2}$ index displays PRESS (the predictive residual sum of squares). The $r \_$pred $^{2}$ index related to the predictive correlation coefficient $\left(r_{-}\right.$pred $\left.^{2}>0.6\right)$ [50] is calculated for the test group and is characterized through Equation (2).

$$
r_{\text {pred }}^{2}=\left(\frac{\mathrm{SD}-\mathrm{PRESS}}{\mathrm{SD}}\right)
$$

In Equation (2), the SD index shows the squared deviation sum for molecules amongst the test group biological activities and training group mean activities [51]. Also, the PRESS index indicates the squared deviation summation amongst actual and predicted activity values for molecules individually in the test group. Based on previous studies [52], If $R^{2}$ is bigger than 0.6 , and $R^{2} \mathrm{cv}\left(Q^{2}\right.$ ) is bigger than $0.5,3 \mathrm{D}-\mathrm{QSAR}$ models are acceptable. The regression model action composed was assessed using the RMSE index. For the data group, RMSE is computed as 


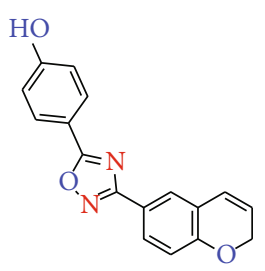

(a)

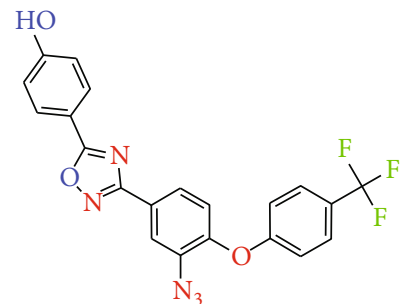

(c)

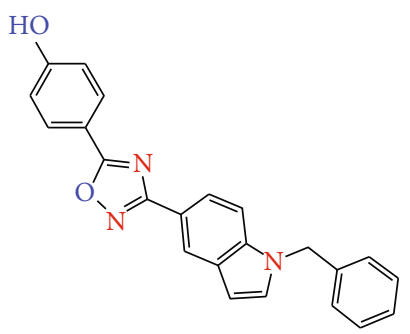

(e)<smiles></smiles>

(b)<smiles>O=S(=O)(c1ccccc1)c1ccc(-c2noc(-c3ccc(O)cc3)n2)cc1</smiles>

(d)

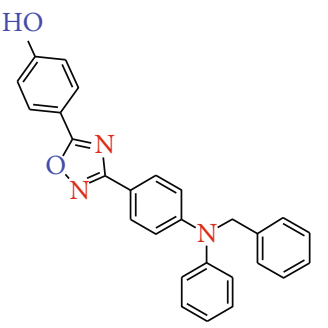

(f)

FIGURE 1: Examples of diverse structure for the 1,2,4-oxadiazole compounds.

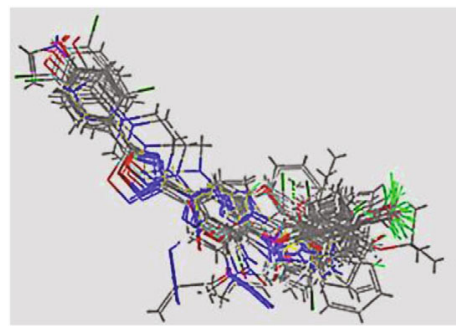

Aligned compounds

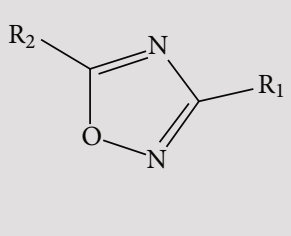

Template
FIGURE 2: 3D-QSAR structure superposition and alignment of the series (Strick model).

Equation (3) [53].

$$
\text { RMSE }=\sqrt{\frac{\sum_{i=1}^{n}\left(y_{i}-y \wedge_{l}\right)^{2}}{n}} .
$$

The 3D-QSAR model with the sixth component of the PLS factor was considered as the best for 1,2,4-oxadiazole derivatives. This model was approved for its precision in the ligand activity estimate in the training group [51]. Scatter plots for experimental and predicted activities of ligands showed a notable linear correlation. In Figures 4(a) and $4(\mathrm{~b})$, the average difference of values of predicted and experimental for training and test groups is exposed, respectively.

2.3. Applicability Domain. APD can be determined using resemblance measures relying on the Euclidean distances between the entire compounds test and training. A comparison between the distance of the test compounds and their nearest neighbor to a predefined threshold in the training group is done, and the prediction is considered inaccurate when the interval is higher than that. The determination of APD was done based on the displayed formula, as follows.

$$
\mathrm{APD}=(d)+\mathrm{Z} \delta .
$$

$\delta$ and $d$ were calculated in a series of steps: first, the mean of Euclidean distances among all training compound pairs was estimated. Then, the collection of distances lower than the median was determined. $\delta$ and $d$ were finally measured as the standard deviation and mean of distances that included in this set. The value equals 0.5 was selected for $Z$ , which was the experimental cutoff in this study. For the applicability domain calculation, we utilized "AD using standardization approach" in DTC Lab (https://dtclab.webs .com/software-tools) [54-56].

$Y$-Randomization Test

The procedure of $Y$-randomization guarantees the validity of a 3D-QSAR model [57]. The dependent changeable 

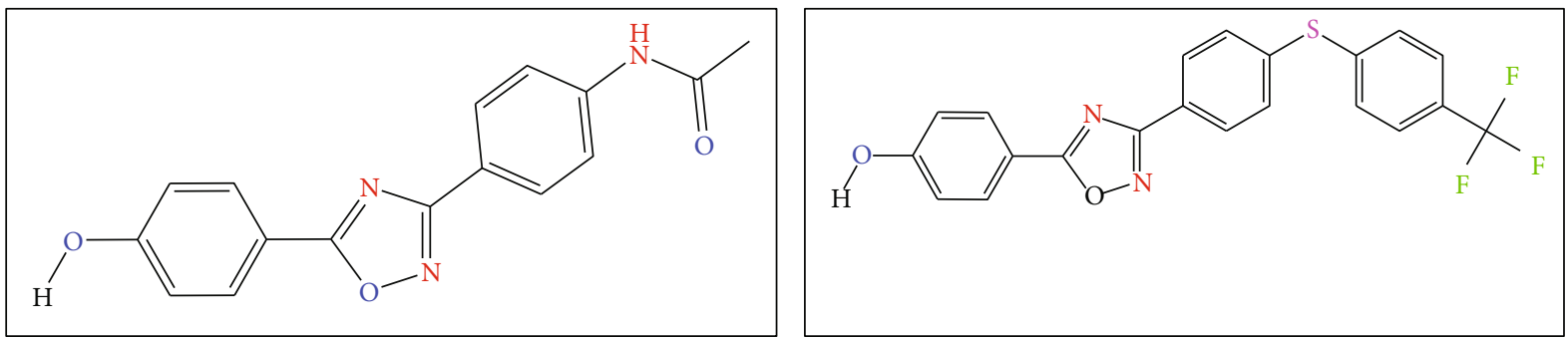

FIgURE 3: (b) Structure of compound $\mathbf{8 9}$ with the best active. (a) Structure of compound $\mathbf{1 2 0}$ with the lowest active.

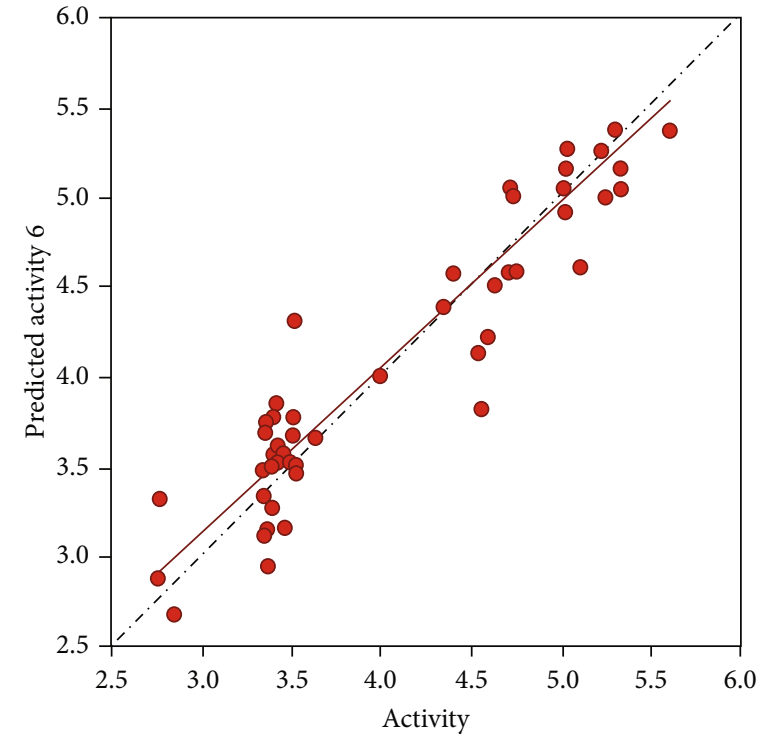

(a)

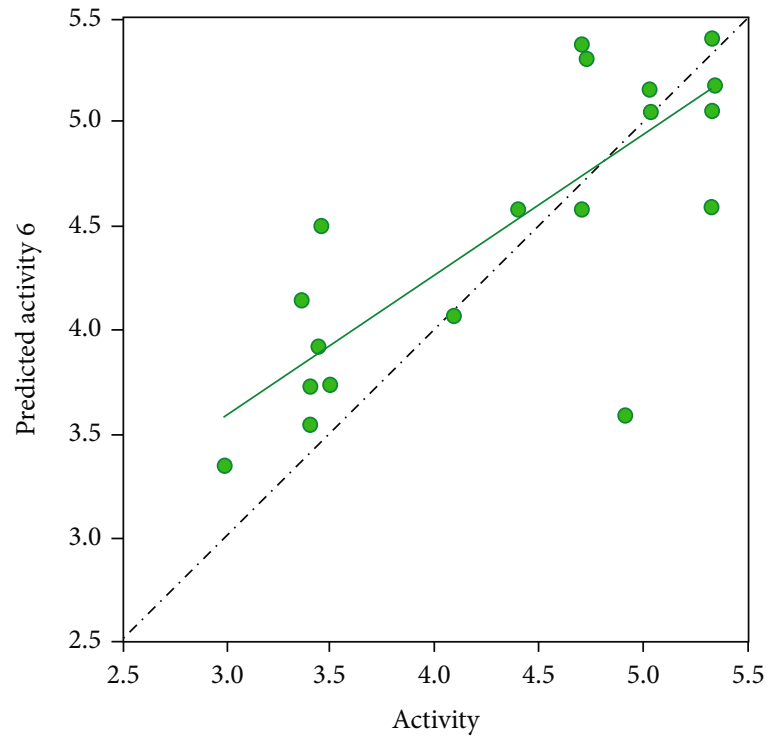

(b)

FIGURE 4: Scatter plot of the observed activity versus predicted activity of (a) training group compounds $\left(y=0.92 x+0.34, R^{2}=0.92\right)$ and $(\mathrm{b})$ test group compounds with the best fit line $\left(y=0.68 x+1.54, R^{2}=0.65\right)$.

vector is altered accidentally, and a novel 3D-QSAR model is produced. The strategy is repeated several times and if the recently produced 3D-QSAR models show low $R^{2}$ and $Q^{2}$ values, the accurateness of the original model is confirmed [58].

2.4. Docking Studies. One most frequent tool for drug design is molecular docking, which employs a mode of association between binding sites of a suitable target with small molecules. Polypeptide structure, SrtA (accession number: $2 \mathrm{KID}$ ), was acquired from the PDB data bank. Here, smallmolecule docking in its active site and its analysis was done via Molecular Operation Environment (MOE) software (http://www.chemcomp.com) for selecting out the most active compound in terms of pMIC ( $\mathrm{pMIC}=2.77)$ with SrtA polypeptide. Before docking, the preparation of the ligand was done, and the 2D structure of ligand was set up by Chemoffice 12.0 which was further changed to $3 \mathrm{D}$ format by Hyper Chem7 software and was optimized employing PM3 semiempirical tool. Also, removal of crystallographic water molecules was done followed by association with $\mathrm{pH} 7$ (for suitable ionization for both alkaline and acidic amino acids) and finally, hydrogen bonds were added. Utilizing the man- ual recommended parameters of the MOE energy minimization with a gradient of 0.05 and MMFF94X ff (force field), the energy of the retrieved protein molecule was calculated. The docking was done with force field as a filtration method via the triangle matcher placement, and the scoring function of the London DG algorithm in combination and the best conformation was analyzed in more details with the LigX module in MOE software. Docking was accomplished for the best compound (compound 89) with the lowest MIC (Figure 5(a)) utilizing the default setting of MOE-Dock [12]. In the last section of the docking process, the selected ligand conformation was further investigated for its interactions of binding. The hydrophilic and hydrophobic field map for compound 89 was also formed (Figure 6(a)). The 2D pictures of the docked conformation of compound 89 are exhibited in Figure 6(b). The compound position in the protein active site is illustrated in Figure 6(c) [59-65].

\section{Results and Discussion}

3.1. 3D-QSAR Model. The 3D-QSAR model was created utilizing PLS regression statistics with the grid spacing $1 \AA$. The seven PLS factors were requested from the program, and the 


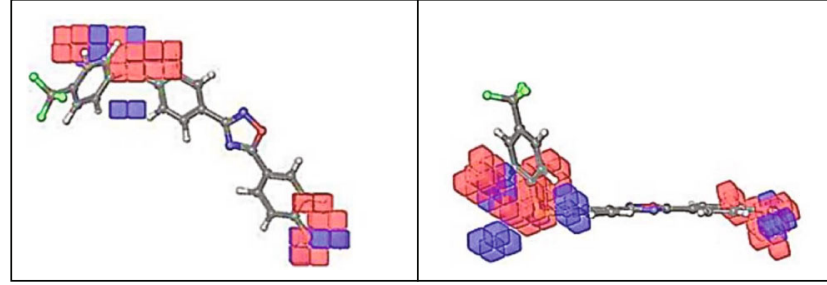

(a)

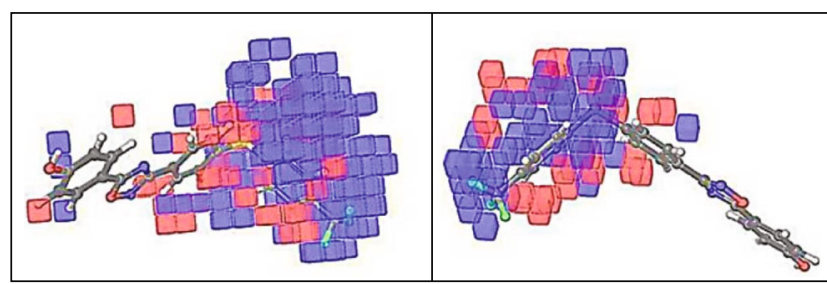

(c)

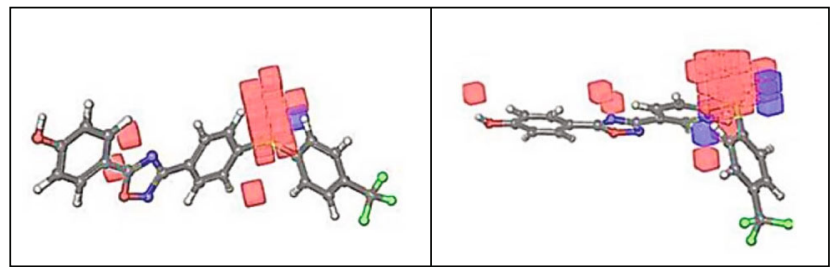

(e)

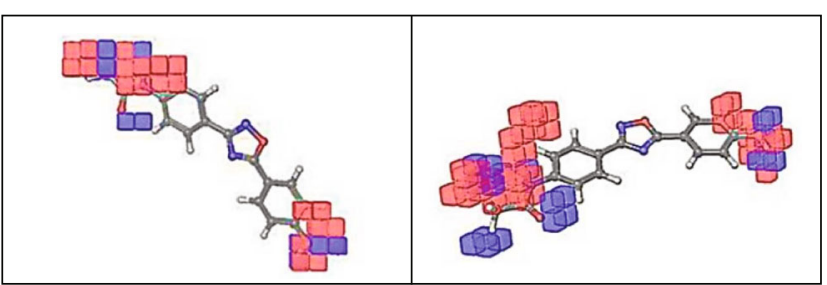

(b)

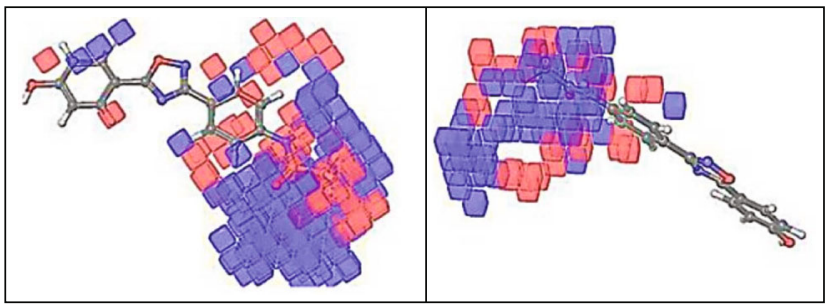

(d)

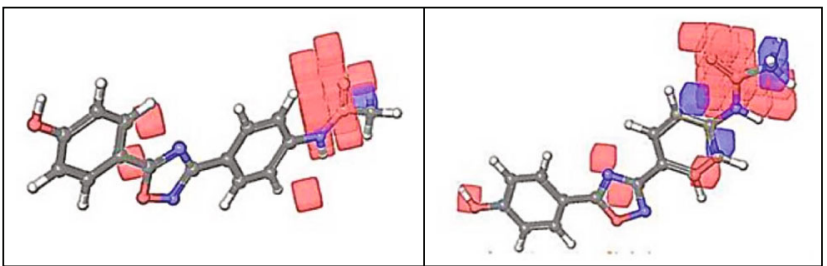

(f)

FIGURE 5: The visualizing of the 3D-QSAR model in the field of unfavorable and favorable effects of hydrogen bond donor (HBD) in: (a) ligand 89 and (b) ligand 120. The visualizing of the 3D-QSAR model in the field of unfavorable and favorable effects of interaction in (c) ligand 89 and (d) ligand 120. The visualizing of the 3D-QSAR model in the field of unfavorable and favorable effects of electronwithdrawing groups in (e) ligand $\mathbf{8 9}$ and (f) ligand 120 in two different dimensions, (positive coefficient color: dark blue, negative coefficient color: red, most active compound is $\mathbf{8 9}(\mathrm{pMIC}=5.617)$, and least active compound is $\mathbf{1 2 0}(\mathrm{pMIC}=2.771)$ ).

best model was observed for the sixth PLS factor owing to its high statistical importance and predictability. (Table 1) The fractions of field and parameters of statistical measured in QSAR-based Gaussian are organized in Table 2.

3.2. Model Validation. Validation of a common pharmacophore model and its prediction relying on active compounds were distinguished by $Q_{\mathrm{cv}}{ }^{2}=0.5479$ (Table 1 ). The training group $R^{2}$ was 0.9235 , which revealed the importance of this model. The produced model stability differs from 0.994 to 0.674 . The value of $F$ was observed to be 179.0. Moreover, a $P$ value equal to $1.95 e-047$ and Pearson $r$ equals 0.8050 showed an assurance of a higher degree in the model. The standard deviation and the root-mean-square error were equal to 0.2291 and 0.48 , respectively, which depicts the strength of the created model in the test for the estimation of the unrecognized compounds. The values of measured pMIC related to the ligands which were included in the predicted group are summarized in Suppl. Table $1 . R^{2}$ values greater than 0.5 as seen amongst the experimental and predicted values produced in the suitable model could show the inhibitory activity that was not included in the progression procedure. [66, 67]. These outcomes suggest that this method can analyze the QSAR model and the ligand-receptor interactions and could be employed in the design of new imidazole inhibitors. Scatter plots, given in Figures 3(a) and 3(b), showed a moderate distinction between the values of two groups, experimental and predicted, and striking linear correlation.

3.3. Applicability Domain. Reports of model constraints by the APD are critical. This shows an important aspect because the user can not only creatively and easily design new compounds but also they can be warned for the estimation validity as to when the structure features cannot be provided via the model. Therefore, after selecting the best model, the ADP of the model showed that the predicted model was valid. In the applicability domain, the compound was completely put inside the range. Indeed, all ligands were in the applicability domain and hence can be assumed as acceptable.

\section{$Y$-Randomization Test}

Further confirmation of the model was done via $Y$-randomization. Ten accidental changes of the $Y$ vector were done, and the low values of $R^{2}$ and $Q^{2}$ were calculated. The range of the $R^{2}$ and $Q^{2}$ values were 0.34 to 0.57 and -0.45 to -0.65 , respectively. It needs to be mentioned that every $Y$ vector random stage was followed by the perfect training method to improve the new QSAR model, involving the choice of the most proper descriptors [68].

3.4. 3D-QSAR Contour Map Analysis. Contour plot interpretation was done to detect the influence of spatial arrangement on the structural characteristics like hydrophobic, 


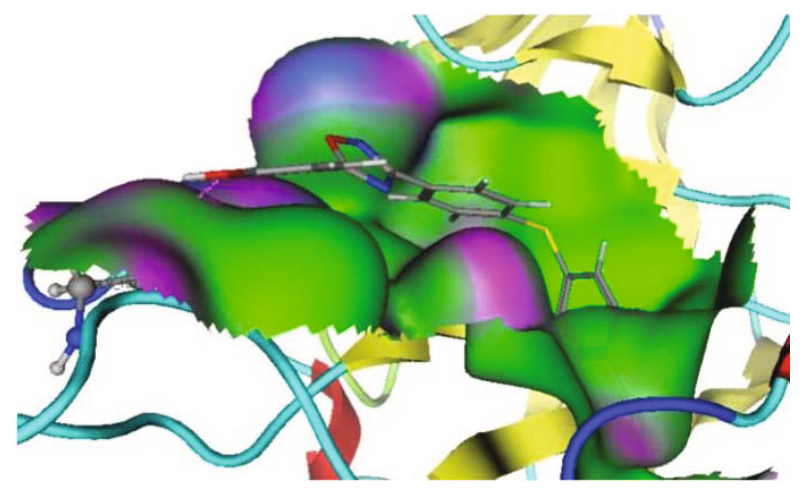

H-bonding:

Hydrophobic:

Mild polar:

(a)
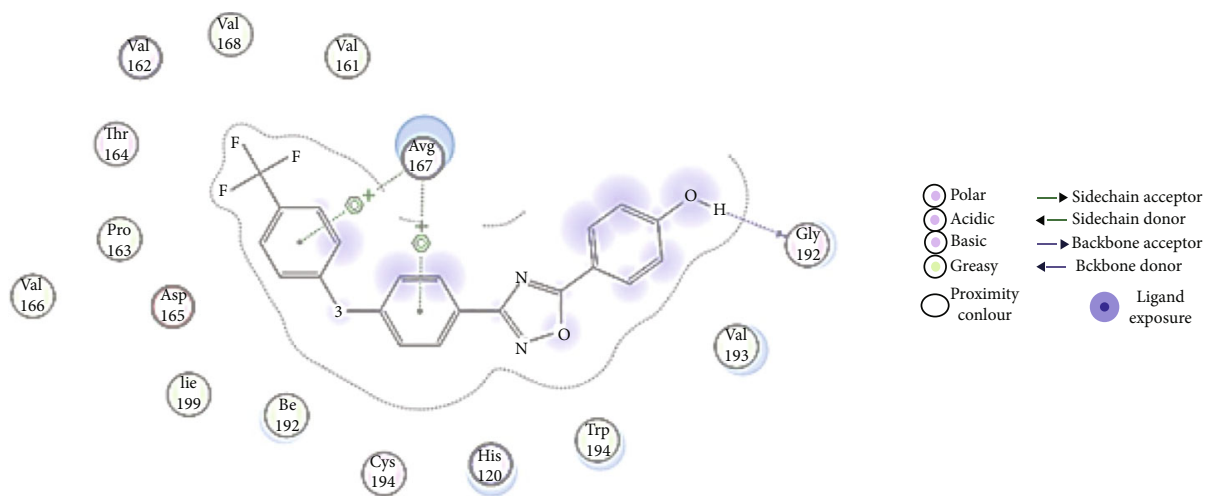

$\begin{array}{ll}\text { Solventresidue } & \text { 回回Arene-arene } \\ \text { Metal complex } & \text { H Arene-H }\end{array}$

Solvent contact $\quad$ O. + Arene-H

(b)

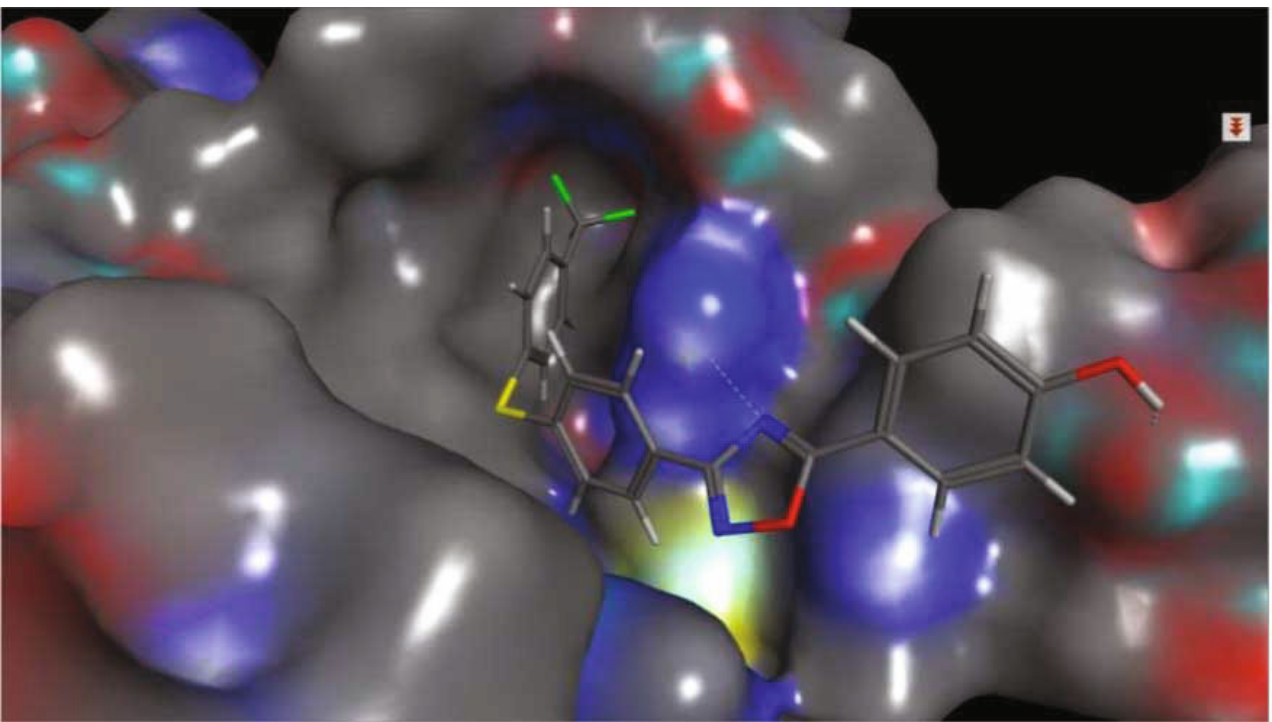

(c)

Figure 6: (a) The display of the image related to hydrophobic and hydrophilic fields for compound 89 into the active site (2KID). (b) The $2 \mathrm{D}$ pictures of the docked conformation of compound 89. (c) The positioning of the compound $\mathbf{8 9}$ in the protein active site.

ionic, electrostatic, H-bond acceptor, and H-bond donor locations on oxadiazole inhibitory effects. The positive contribution appeared in blue-colored cubes, and the negative contribution was visible in red. Figures 5(a)-5(f) are shown for the identification of the acceptable and unacceptable important interactions in two different dimensions, which resulted in the use of the QSAR model. HBD nature comparison of compound 89 (the best activity, Figure 5(a)) and the compound 120 (the least activity, Figure 5(b)) displays unacceptable and acceptable regions as red and blue cubes, respectively. Hydrogen bond donor maps showed that unfavorable locations placed next to the nitrogen atom of amide present on one side of the oxadiazole (Figure 5(a)) are an HBD group which is in the inappropriate place, whereas for the most active molecule, unfavorable regions lay near the sulfur atom which is not an HBD group (Figure 5(b)). 
TABLE 1: PLS statistical parameters of the model QSAR model.

\begin{tabular}{lcccccccc}
\hline PLS & SD & $R^{2}$ & $F$ & $P$ & Stability & RMSE & $Q^{2}$ & Pearson- $R$ \\
\hline 1 & 0.4285 & 0.7171 & 238.3 & $1.62 e-027$ & 0.994 & 0.45 & 0.6744 \\
2 & 0.3774 & 0.7830 & 167.8 & $1.41 e-031$ & 0.971 & 0.48 & 0.6323 \\
3 & 0.3254 & 0.8403 & 161.4 & $1.57 e-036$ & 0.907 & 0.44 & 0.6900 \\
4 & 0.2808 & 0.8824 & 170.7 & $2.1 e-041$ & 0.835 & 0.43 & 0.7056 \\
5 & 0.2503 & 0.9076 & 176.8 & $5.91 e-045$ & 0.773 & 0.45 & 0.6772 \\
6 & 0.2291 & 0.9235 & 179.0 & $1.95 e-047$ & 0.722 & 0.48 & 0.6319 & 0.8462 \\
7 & 0.2070 & 0.9382 & 190.9 & $2.31 e-050$ & 0.674 & 0.49 & 0.6134 \\
\hline
\end{tabular}

SD: standard deviation of regression; $R^{2}$ : regression coefficient; $F$ : variance ratio (ratio of the model variance to the observed activity variance); $P$ : significance level of variance ratio; $Q^{2}$ : Crossvalidated correlation coefficient for the test group; RMSE: the RMS error in the test group predictions; Pearson- $R$ : correlation among the predicted and observed activity for the test group.

TABLE 2: Seven factors of PLS were calculated for the QSAR model.

\begin{tabular}{lccccc}
\hline \# factors & H-bond donor & Hydrophobic/nonpolar & Negative ionic & Positive ionic & Electron-withdrawing \\
\hline 1 & 0.028420 & 0.709533 & 0.030517 & 0.031581 & 0.156872 \\
2 & 0.029319 & 0.646792 & 0.039072 & 0.039833 & 0.190441 \\
3 & 0.028610 & 0.647774 & 0.045189 & 0.045139 & 0.200709 \\
4 & 0.029662 & 0.668252 & 0.038533 & 0.037152 & 0.195964 \\
5 & 0.029213 & 0.666539 & 0.039458 & 0.037827 & 0.195301 \\
6 & 0.029931 & 0.669972 & 0.037575 & 0.035876 & 0.194004 \\
7 & 0.032992 & 0.677689 & 0.033050 & 0.032073 & 0.191071 \\
\hline
\end{tabular}

Also, the hydrogen of the hydroxyl group on p-hydroxyphenyl, present on the other side of the oxadiazole near the desirable region, is available for two compounds-categorized as active and the least active.

Compounds such as ligand 89 with $p-\mathrm{CF}_{3}-$ phenylthio hydrophobic substituent had higher potency values than compounds without substitute mentioned such as ligand 120, because of the presence of favorable hydrophobic regions in that position (Figures 5(c) and 5(d)), which was confirmed by the results obtained from previous CoMFA studies [69]. For less active ligands such as compound 120, the hydrophilic group (amid) fell into the favorable hydrophobic envelope that is not suitable for the hydrophilic groups. Comparison between the effects of the electron-withdrawing moieties of the best compound $\mathbf{8 9}$ with an electron p-CF3-phenylthio group and the least active compound 120 with the acetamido group was shown in Figures 5(f) and 5(e).

3.5. Docking Studies. The MOE-Dock program was utilized to check the stability of the models created in this study with the sortase A polypeptide receiver (PDB code 2KID). Studies of docking showed that interactions were commanded by aromaticity and hydrophobicity due to the position of phenol moiety (Figures 6(a)-6(c)). The best compound ( $\mathrm{pMIC}=5.617$ ) was connected into the binding cavity of polypeptide SrtA with high affinity and created interactions in association with the oxygen of phenol with the Gly192 residue in one side of the ligand, while two rings on the other side of the oxadiazole have two interactions arene-cation with Arg 197 residue. The scores of docking studies of the best compound were $-11.12 \mathrm{kcal} / \mathrm{mol}$. Therefore, the com- pound 89 had a three-point attachment with the protein binding cavity. The interactions were present in the region containing Gly 192 and Arg 197 residues (Figure 6(b)). In general, oxygen is bound to hydrogen of hydroxyl in the acidic part of Gly 192 residue that showed only one hydrogen bond. This subject is visible by analyzing the hydrophilic and hydrophobic regions of compound 89 (Figure 6(a)). 3DQSAR contour map analysis studies confirm this and showed that the compounds like ligand $\mathbf{8 9}$ are placed in a hydrophobic envelope (Figure 6(c)).

\section{Conclusions}

Using model prediction by 3D-QSAR studies of 120 analogs of 1,2,4-oxadiazoles and docking, we provided insights into the critical features needed for the design of inhibitors of SrtA. 3D-QSAR modeling was performed to provide a structural network for the comprehension of structure-activity relationships of the ligands present in the study. Studies of molecular docking were done to create desirable poses that bind to these compounds. The gets scores in VS (virtual screening) of compounds gave us chemically important points for the design and improvement of novel oxadiazoles as sortase inhibitors. The most active compound (89) of 1,2,4-oxadiazoles used in this study had four rings, named $\mathrm{A}, \mathrm{B}, \mathrm{C}$, and D. HBD moieties in the A ring were essential for antibacterial activity. The aniline, phenol, and some heterocyclic compounds with hydrogen-bonding ability, such as pyrazoles, were allowed. These findings are in line with previous results. In line with previous explanations on the 3D-QSAR map analysis section, a hydrophobic substituent 
was seen essential for the activity in the $\mathrm{D}$ ring region [24, 69]. In general, by $3 \mathrm{D}-\mathrm{QSAR}$, we attempted to study the structural diversity in the ring $\mathrm{D}$ antibacterial activity in a 1,2,4-oxadiazoles region (Figure 1). Finally, our findings suggest that the 1,2,4-oxadiazoles are inhibitors of sortase $\mathrm{A}$ and act against $S$. aureus, further holding great promising potential as future therapeutics for treating hospital infections.

\section{Abbreviations}

$R^{2}: \quad$ Regression coefficient

$Q^{2}: \quad$ Crossvalidation correlation coefficient

APD: Applicability domain

PLS: Partial least square

SD: $\quad$ Standard deviation

RMSD: Root mean square deviation

RMSE: Root mean squared error

HBD: Hydrogen-bond donor.

\section{Data Availability}

The data about this original article are available upon reasonable request.

\section{Conflicts of Interest}

The authors confirm they have no conflict of interest.

\section{Supplementary Materials}

Supplementary Table 1: molecular view, assay-organism and assay-strain, the experimental, and predicted corresponding values of pMIC gained via QSAR models. (Supplementary Materials)

\section{References}

[1] L. Si, P. Li, X. Liu, and L. Luo, "Chinese herb medicine against sortase a catalyzed transformations, a key role in gram-positive bacterial infection progress," Journal of Enzyme Inhibition and Medicinal Chemistry., vol. 31, supplement 1, pp. 184-196, 2016.

[2] C. Garandeau, H. Réglier-Poupet, I. Dubail, J.-L. Beretti, P. Berche, and A. Charbit, "The sortase SrtA of listeria monocytogenes is involved in processing of internalin and in virulence," Infection and Immunity., vol. 70, no. 3, pp. 13821390, 2002.

[3] Y. Zong, T. W. Bice, H. Ton-That, O. Schneewind, and S. V. Narayana, "Crystal structures of_Staphylococcus aureus_sortase A and its substrate complex," Journal of Biological Chemistry., vol. 279, no. 30, pp. 31383-31389, 2004.

[4] A. Grigoletto, K. Maso, and G. Pasut, Enzymatic Approaches to New Protein Conjugates, Elsevier, Polymer-Protein Conjugates, 2020.

[5] H. M. Burke, L. McSweeney, and E. M. Scanlan, "Exploring chemoselective _S_-to- _N $\mathrm{N}_{-}$acyl transfer reactions in synthesis and chemical biology," Nature Communications, vol. 8, no. 1, pp. 1-16, 2017.

[6] S. Cascioferro, D. Raffa, B. Maggio, M. V. Raimondi, D. Schillaci, and G. Daidone, "Sortase a inhibitors: recent advances and future perspectives," Journal of Medicinal Chemistry., vol. 58, no. 23, 2015.

[7] J. Wang, H. Li, J. Pan et al., "Oligopeptide targeting Sortase a as potential anti-infective therapy for Staphylococcus aureus," Frontiers in Microbiology., vol. 9, p. 245, 2018.

[8] M. V. Raimondi, R. Listro, M. G. Cusimano et al., "Novel sortase A inhibitors to counteract gram-positive bacterial biofilms," Multidisciplinary Digital Publishing Institute Proceedings., vol. 22, no. 1, 2019.

[9] B. T. Russo, Y. A. Ayinuola, D. Singh et al., "The M protein of streptococcus pyogenes strain AP53 retains cell surface functional plasminogen binding after inactivation of the sortase A Gene," Journal of Bacteriology., vol. 202, no. 10, 2020.

[10] L. A. Marraffini, A. C. DeDent, and O. Schneewind, "Sortases and the art of anchoring proteins to the envelopes of grampositive bacteria," Microbiology and Molecular Biology Reviews, vol. 70, no. 1, pp. 192-221, 2006.

[11] Y. Zong, S. K. Mazmanian, O. Schneewind, and S. V. Narayana, "The structure of sortase B, a cysteine transpeptidase that tethers surface protein to the _Staphylococcus aureus_ Cell Wall," Structure, vol. 12, no. 1, pp. 105-112, 2004.

[12] Y. Guo, S. Cai, G. Gu, Z. Guo, and Z. Long, "Recent progress in the development of sortase A inhibitors as novel anti-bacterial virulence agents," RSC Advances., vol. 5, no. 62, pp. 4988049889, 2015.

[13] C.-Y. Kang, I.-H. Huang, C.-C. Chou et al., "Functional analysis of_Clostridium difficile_sortase B reveals key residues for catalytic activity and substrate specificity," Journal of Biological Chemistry., vol. 295, no. 11, pp. 3734-3745, 2020.

[14] T. Huang, Z. Peng, M. Hu et al., "Interactions between_Lactobacillus plantarum_ NCU116 and its environments based on extracellular proteins and polysaccharides prediction by comparative analysis," Genomics, vol. 112, no. 5, pp. 3579-3587, 2020.

[15] H. Bierne and P. Cossart, "Listeria monocytogenes surface proteins: from genome predictions to function," Microbiology and Molecular Biology Reviews, vol. 71, no. 2, pp. 377-397, 2007.

[16] S. K. Mazmanian, H. Ton-That, and O. Schneewind, "Sortasecatalysed anchoring of surface proteins to the cell wall of Staphylococcus aureus," Molecular Microbiology., vol. 40, no. 5, pp. 1049-1057, 2001.

[17] T. Hampton, "Report reveals scope of US antibiotic resistance threat," Journal of the American Medical Association, vol. 310, no. 16, pp. 1661-1663, 2013.

[18] J.-Y. Maillard, S. F. Bloomfield, P. Courvalin et al., "Reducing antibiotic prescribing and addressing the global problem of antibiotic resistance by targeted hygiene in the home and everyday life settings: a position paper," American Journal of Infection Control., vol. 48, no. 9, pp. 1090-1099, 2020.

[19] Z. M. Burcham, C. J. Schmidt, J. L. Pechal et al., "Detection of critical antibiotic resistance genes through routine microbiome surveillance," PLoS One, vol. 14, no. 3, article e0213280, 2019.

[20] F. Cunha, J. Nogueira, and A. de Aguiar, "Synthesis and antibacterial evaluation of 3,5-diaryl-1, 2, 4-oxadiazole derivatives," Journal of the Brazilian Chemical Society, vol. 29, no. 11, pp. 2405-2416, 2018.

[21] J. Lee, S. Cho, and M.-h. Kim, "Discovery of CNS-like D3Rselective antagonists using 3D pharmacophore guided virtual screening," Molecules, vol. 23, no. 10, p. 2452, 2018. 
[22] R. R. Watkins, M. Z. David, and R. A. Salata, "Current concepts on the virulence mechanisms of meticillin-resistant Staphylococcus aureus," Journal of Medical Microbiology., vol. 61, no. 9, pp. 1179-1193, 2012.

[23] L. García-Álvarez, M. T. G. Holden, H. Lindsay et al., "Meticillin-resistant_Staphylococcus aureus_with a novel _mecA_ homologue in human and bovine populations in the UK and Denmark: a descriptive study," The Lancet Infectious Diseases., vol. 11, no. 8, pp. 595-603, 2011.

[24] D. Ding, M. A. Boudreau, E. Leemans et al., "Exploration of the structure-activity relationship of 1,2,4-oxadiazole antibiotics," Bioorganic \& Medicinal Chemistry Letters., vol. 25, no. 21, pp. 4854-4857, 2015.

[25] I. Gould, J. Reilly, D. Bunyan, and A. Walker, "Costs of healthcare-associated methicillin-resistant _Staphylococcus aureus_and its control," Clinical Microbiology and Infection., vol. 16, no. 12, pp. 1721-1728, 2010.

[26] Z. Tavajjohi, R. Moniri, and A. Khorshidi, "Detection and characterization of multidrug resistance and extended-spectrum-beta-lactamase-producing (ESBLS) Pseudomonas aeruginosa isolates in teaching hospital," African Journal of Microbiology Research., vol. 5, no. 20, pp. 3223-3228, 2011.

[27] A. S. Kaka, A. M. Rueda, S. A. Shelburne III, K. Hulten, R. J. Hamill, and D. M. Musher, "Bactericidal activity of orally available agents against methicillin-resistant Staphylococcus aureus," Journal of Antimicrobial Chemotherapy., vol. 58, no. 3, pp. 680-683, 2006.

[28] J. Janardhanan, J. E. Meisel, D. Ding et al., "In vitro and in vivo synergy of the oxadiazole class of antibacterials with $\beta$-lactams," Antimicrobial Agents and Chemotherapy., vol. 60, no. 9, pp. 5581-5588, 2016.

[29] R. McCool, I. M. Gould, J. Eales et al., "Systematic review and network meta-analysis of tedizolid for the treatment of acute bacterial skin and skin structure infections caused by MRSA," BMC Infectious Diseases., vol. 17, no. 1, p. 39, 2017.

[30] S. Ningaiah, U. K. Bhadraiah, S. Keshavamurthy, and C. Javarasetty, "Novel pyrazoline amidoxime and their 1,2,4oxadiazole analogues: Synthesis and pharmacological screening," Bioorganic \& Medicinal Chemistry Letters., vol. 23, no. 16, pp. 4532-4539, 2013.

[31] P. Dadgostar, "Antimicrobial resistance: implications and costs," Infection and Drug Resistance., vol. Volume 12, pp. 3903-3910, 2019.

[32] R. J. Loureiro, F. Roque, A. T. Rodrigues, M. T. Herdeiro, and E. Ramalheira, "Use of antibiotics and bacterial resistances: brief notes on its evolution," Revista Portuguesa de Saúde Pública., vol. 34, no. 1, pp. 77-84, 2016.

[33] R. Musumeci, C. E. A. Cocuzza, C. G. Fortuna, P. Andrea, and A. P. PICCIONELLO, "4-Oxadiazol compounds active against gram-positive pathogens,” Google Patents, vol. 1, 2018.

[34] C. G. Fortuna, C. Bonaccorso, A. Bulbarelli et al., "New linezolid-like 1,2,4-oxadiazoles active against Gram-positive multiresistant pathogens," European Journal of Medicinal Chemistry., vol. 65, pp. 533-545, 2013.

[35] E. Spink, D. Ding, Z. Peng et al., "Structure-activity relationship for the oxadiazole class of antibiotics," Journal of Medicinal Chemistry., vol. 58, no. 3, pp. 1380-1389, 2015.

[36] M. A. Boudreau, D. Ding, J. E. Meisel et al., "Structure-activity relationship for the oxadiazole class of antibacterials," ACS Medicinal Chemistry Letters., vol. 11, no. 3, pp. 322-326, 2020.
[37] E. A. Hall, Fighting Bacterial Drug Resistance by Targeting Peptidoglycan Biosynthesis: Depsipeptide Antibiotics and Biological Probes, Indiana University, 2014.

[38] S. Yatam, R. Gundla, S. S. Jadav et al., "Focused library design and synthesis of 2-mercapto benzothiazole linked 1,2,4-oxadiazoles as COX-2/5-LOX inhibitors," Journal of Molecular Structure., vol. 1159, pp. 193-204, 2018.

[39] P. Zhang, J.-H. Chen, X. Dong et al., "6r, a novel oxadiazole analogue of ethacrynic acid, exhibits antitumor activity both in vitro and in vivo by induction of cell apoptosis and Sphase arrest," Biomedicine \& Pharmacotherapy., vol. 67, no. 1, pp. 58-65, 2013.

[40] S. Cascioferro and D. Schillaci, "The future of antibiotic: from the magic bullet to the smart Bullet," Technology, vol. 6, no. 5, 2014.

[41] S. E. Adeniji, S. Uba, and A. Uzairu, "Theoretical modeling and molecular docking simulation for investigating and evaluating some active compounds as potent anti-tubercular agents against MTB CYP121 receptor," Future Journal of Pharmaceutical Sciences., vol. 4, no. 2, pp. 284-295, 2018.

[42] A. Khatkar, A. Nanda, P. Kumar, and B. Narasimhan, "Synthesis, antimicrobial evaluation and QSAR studies of _p-_ coumaric acid derivatives," Arabian Journal of Chemistry., vol. 10, pp. S3804-S3815, 2017.

[43] J. J. van Zanden, H. M. Wortelboer, S. Bijlsma et al., "Quantitative structure activity relationship studies on the flavonoid mediated inhibition of multidrug resistance proteins 1 and 2," Biochemical Pharmacology, vol. 69, no. 4, pp. 699-708, 2005.

[44] K. E. Hevener, D. M. Ball, J. K. Buolamwini, and R. E. Lee, "Quantitative structure-activity relationship studies on nitrofuranyl anti- tubercular agents," Bioorganic \& Medicinal Chemistry., vol. 16, no. 17, pp. 8042-8053, 2008.

[45] M. F. Khan, G. Verma, W. Akhtar et al., "Pharmacophore modeling, 3D-QSAR, docking study and ADME prediction of acyl 1,3,4-thiadiazole amides and sulfonamides as antitubulin agents," Arabian Journal of Chemistry., vol. 12, no. 8, pp. 5000-5018, 2019.

[46] B. Rasulev, "Recent developments in 3D QSAR and molecular docking studies of organic and Nanostructures," Chemistry, vol. 2133, 2017.

[47] N. Shakour, R. Cabezas, J. G. Santos et al., "Curcumin can bind and interact with CRP: an in silico study," Pharmacological Properties of Plant-Derived Natural Products and Implications for Human Health., vol. 1308, pp. 91-100, 2021.

[48] N. Shakour, A. Sahebkar, G. Karimi et al., "Design, synthesis and biological evaluation of novel 5-(imidazolyl-methyl) thiazolidinediones as antidiabetic agents," Bioorganic Chemistry, vol. 115, article 105162, 2021.

[49] N. Chirico and P. Gramatica, "Real external predictivity of QSAR models: how to evaluate it? Comparison of different validation criteria and proposal of using the concordance correlation coefficient," Journal of Chemical Information and Modeling., vol. 51, no. 9, pp. 2320-2335, 2011.

[50] A. I. Foudah, A. A. Sallam, M. R. Akl, and K. A. El Sayed, "Optimization, pharmacophore modeling and 3D-QSAR studies of sipholanes as breast cancer migration and proliferation inhibitors," European Journal of Medicinal Chemistry., vol. 73, pp. 310-324, 2014.

[51] Y. Xu, Z. He, H. Liu et al., "3D-QSAR, molecular docking, and molecular dynamics simulation study of thieno $[3,2-b]$ 
pyrrole-5-carboxamide derivatives as LSD1 inhibitors," RSC Advances., vol. 10, no. 12, pp. 6927-6943, 2020.

[52] S. Norouzia, M. Farahania, and S. N. Ebrahimi, "The integration of pharmacophore-based 3D QSAR modeling and virtual screening in identification of natural product inhibitors against SARS-CoV-2," Pharmaceutical Sciences., vol. 27, pp. S94-S108, 2021.

[53] A. Rácz, D. Bajusz, and K. Héberger, “Consistency of QSAR models: correct split of training and test sets, ranking of models and performance parameters," SAR and QSAR in Environmental Research., vol. 26, no. 7-9, pp. 683-700, 2015.

[54] K. Roy, S. Kar, and P. Ambure, "On a simple approach for determining applicability domain of QSAR models," Chemometrics and Intelligent Laboratory Systems, vol. 145, pp. 2229, 2015.

[55] H. Hadni and M. Elhallaoui, "3D-QSAR, docking and ADMET properties of aurone analogues as antimalarial agents," Heliyon., vol. 6, no. 4, article e03580, 2020.

[56] A. A. Alizadeh, B. Jafari, and S. Dastmalchi, "Alignment independent 3D-QSAR studies and molecular dynamics simulations for the identification of potent and selective $\mathrm{S}_{1} \mathrm{P}_{1}$ receptor agonists," Journal of Molecular Graphics and Modelling., vol. 94, article 107459, 2020.

[57] M. Shen, C. Béguin, A. Golbraikh, J. P. Stables, H. Kohn, and A. Tropsha, "Application of predictive QSAR models to database mining: identification and experimental validation of novel anticonvulsant compounds," Journal of Medicinal Chemistry., vol. 47, no. 9, pp. 2356-2364, 2004.

[58] Z. Ul-Haq, U. Mahmood, and S. Reza, “A combined 3D-QSAR and molecular docking strategy to understand the binding mechanism of V600E B-RAF inhibitors," Molecular Diversity., vol. 16, no. 4, pp. 771-785, 2012.

[59] N. K. Salam, R. Nuti, and W. Sherman, "Novel method for generating structure-based pharmacophores using energetic analysis," Journal of Chemical Information and Modeling., vol. 49, no. 10, pp. 2356-2368, 2009.

[60] V. Sharma, H. Kumar, and S. Wakode, "Pharmacophore generation and atom based 3D-QSAR of quinoline derivatives as selective phosphodiesterase 4B inhibitors," RSC Advances., vol. 6, no. 79, pp. 75805-75819, 2016.

[61] M. Athar, M. Y. Lone, V. M. Khedkar, and P. C. Jha, "Pharmacophore model prediction, 3D-QSAR and molecular docking studies on vinyl sulfones targeting Nrf2-mediated gene transcription intended for anti-Parkinson drug design," Journal of Biomolecular Structure and Dynamics., vol. 34, no. 6, pp. 1282-1297, 2016.

[62] D. Panigrahi, A. Mishra, and S. K. Sahu, "Pharmacophore modelling, QSAR study, molecular docking and insilico ADME prediction of 1, 2, 3-triazole and pyrazolopyridones as DprE1 inhibitor antitubercular agents," SN Applied Sciences., vol. 2, no. 5, pp. 1-28, 2020.

[63] C. N. Cavasotto and M. G. Aucar, "High-throughput docking using quantum mechanical Scoring," Chemistry, vol. 8, 2020.

[64] W.-L. Ye, C. Shen, G.-L. Xiong et al., "Improving dockingbased virtual screening ability by integrating multiple energy auxiliary terms from molecular docking scoring," Journal of Chemical Information and Modeling., vol. 60, no. 9, pp. 4216-4230, 2020.

[65] S. Mirzaei, F. Hadizadeh, F. Eisvand, F. Mosaffa, and R. Ghodsi, "Synthesis, structure-activity relationship and molecular docking studies of novel quinoline-chalcone hybrids as potential anticancer agents and tubulin inhibitors," Journal of Molecular Structure., vol. 1202, article 127310, 2020.

[66] G. Verma, M. F. Khan, W. Akhtar et al., "Pharmacophore modeling, 3D-QSAR, docking and ADME prediction of quinazoline based EGFR inhibitors," Arabian Journal of Chemistry., vol. 12, no. 8, pp. 4815-4839, 2019.

[67] A. Golbraikh and A. Tropsha, "Beware of q2!," Journal of Molecular Graphics and Modelling., vol. 20, no. 4, pp. 269276, 2002.

[68] A. B. Umar, A. Uzairu, G. A. Shallangwa, and S. Uba, "Design of potential anti-melanoma agents against SK-MEL-5 cell line using QSAR modeling and molecular docking methods," $S N$ Applied Sciences., vol. 2, no. 5, pp. 1-18, 2020.

[69] E. Leemans, K. V. Mahasenan, M. Kumarasiri et al., “Threedimensional QSAR analysis and design of new 1,2,4-oxadiazole antibacterials," Bioorganic \& Medicinal Chemistry Letters., vol. 26, no. 3, pp. 1011-1015, 2016. 